\title{
Rademacher functions in BMO
}

\author{
by \\ Sergey V. Astashkin (Samara), Mikhail Leibov (New York) \\ and LECH MALigRANDA (Luleå)
}

\begin{abstract}
The Rademacher sums are investigated in the $B M O$ space on $[0,1]$. They span an uncomplemented subspace, in contrast to the dyadic $B M O_{d}$ space on $[0,1]$, where they span a complemented subspace isomorphic to $l_{2}$. Moreover, structural properties of infinite-dimensional closed subspaces of the span of the Rademacher functions in $B M O$ are studied and an analog of the Kadec-Pełczyński type alternative with $l_{2}$ and $c_{0}$ spaces is proved.
\end{abstract}

1. Introduction. In 1961, when studying some problems concerning partial differential equations, F. John and L. Nirenberg introduced the space $B M O$ of functions of bounded mean oscillation. In 1971 Fefferman [6] announced that the dual to the real Hardy space $H^{1}$ on $\mathbb{R}^{n}$ is $B M O$. Next year, the proof was published by Fefferman and Stein in their paper [7, Theorem 2] (see also Garnett [8, Theorem 4.4], Grafakos [12, Theorem 7.2.2], Kashin and Saakyan [15, Theorem 5.5], and Stein [30, pp. 142-144]). This duality result of Fefferman called considerable attention to the $B M O$ space and after 1971 many results were proved about this space (see e.g. Garnett [8, Chapter VI], Grafakos [12, Chapter 7] and Stein [30, Chapter IV]).

There is also a larger dyadic counterpart $B M O_{d}$ of the space of functions of bounded mean oscillation, $B M O_{d} \supsetneq B M O$. This dyadic space related to $B M O$ was studied already by Garnett and Jones [9]. Since $B M O$ is translation invariant and $B M O_{d}$ is not, $B M O$ is more important in analysis. On the other hand, it is much easier to work with $B M O_{d}$ because of the fact that dyadic cubes are nested (if two open dyadic cubes intersect then one of them is contained in the other).

Consider the Rademacher functions on $[0,1]$ defined by

$$
r_{k}(t)=\operatorname{sign}\left[\sin \left(2^{k} \pi t\right)\right], \quad k \in \mathbb{N}, t \in[0,1],
$$

2010 Mathematics Subject Classification: 46E30, 46B20, 46B42.

Key words and phrases: Rademacher functions, $B M O$ space, dyadic $B M O$ space, subspaces, complemented subspaces. 
and the set of Rademacher sums

$$
R_{n}(t)=\sum_{k=1}^{n} a_{k} r_{k}(t), \quad a_{k} \in \mathbb{R}, \text { for } k=1, \ldots, n \text { and } n \in \mathbb{N} .
$$

The behaviour of Rademacher sums in the spaces $L_{p}=L_{p}[0,1]$ is well known and it is described by the classical Khintchine inequalities: there exist constants $A_{p}, B_{p}>0$ such that for every sequence $\left\{a_{k}\right\}_{k=1}^{n}$ of real numbers and any $n \in \mathbb{N}$ we have

$$
A_{p}\left(\sum_{k=1}^{n}\left|a_{k}\right|^{2}\right)^{1 / 2} \leq\left\|R_{n}\right\|_{L_{p}[0,1]} \leq B_{p}\left(\sum_{k=1}^{n}\left|a_{k}\right|^{2}\right)^{1 / 2}, \quad 0<p<\infty .
$$

Hence, the Rademacher functions $\left\{r_{n}\right\}$ span an isomorphic copy of $l_{2}$ in $L_{p}$ for every $0<p<\infty$. Moreover, the subspace $\left[r_{n}\right]_{n=1}^{\infty}$ is complemented in $L_{p}$ for $1<p<\infty$ and it is not complemented in $L_{1}$ since no complemented infinite-dimensional subspace of $L_{1}$ can be reflexive. In $L_{\infty}$ we have $\left\|R_{n}\right\|_{L_{\infty}[0,1]}=\sum_{k=1}^{n}\left|a_{k}\right|$ and so the Rademacher functions span an isometric copy of $l_{1}$, which is known to be uncomplemented in $L_{\infty}$. Investigations of Rademacher sums in general symmetric (rearrangement invariant) spaces are well presented in the books by Lindenstrauss-Tzafriri [21], Krel̆-Petunin-Semenov [17] and Astashkin [2], where also the definition and several properties of symmetric spaces can be found.

The purpose of this paper is to investigate sums of Rademacher functions in the $B M O$ space on $[0,1]$. Some results are contained in Leibov's dissertation [18] (Proposition 2 with some estimates of type (7), which we correct in this paper, and partly Theorem 5) but, in fact, they are not known to a wide audience since they were not published in any journal and the dissertation is not available.

The paper is organized as follows: after the introduction in Section 1, we collect some necessary definitions, notation, and auxiliary results in Section 2. The main result in Section 3 is Theorem 2 describing the behaviour of Rademacher sums in $B M O[0,1]$. In Section 4 we discuss the complementability of Rademacher subspaces $\mathcal{R}_{d}$ and $\mathcal{R}$ in $B M O_{d}$ and in $B M O$, respectively. Namely, it is well-known that $\mathcal{R}_{d}$ is complemented in $B M O_{d}$ (Theorem 3). At the same time we prove that $\mathcal{R}$ is not complemented in $B M O$ (Theorem 4). Finally, in Section 5, we investigate the structure of infinite-dimensional subspaces of $\mathcal{R}$. In particular, in Theorem 5 we state the following analogue of the Kadec-Pełczyński type alternative for $\mathcal{R}$ : every infinite-dimensional closed subspace $X \subset \mathcal{R}$ is either isomorphic to $l_{2}$ and complemented in $B M O$, or contains a subspace $Y$ isomorphic to $c_{0}$ and complemented in $\mathcal{R}$. Then, in Examples 1 and 2, we construct block bases of the Rademacher system whose span is $l_{2}$ and $c_{0}$, respectively. 
2. Preliminaries and auxiliary results. For any function $f \in L_{1}[0,1]$ and arbitrary interval $I \subset[0,1]$ we denote

$$
f_{I}=\frac{1}{|I|} \int_{I} f(s) d s
$$

where $|I|$ is the Lebesgue measure of $I$. Then, as usual, the space $B M O=$ $B M O[0,1]$ consists of all $f \in L_{1}[0,1]$ such that

$$
\|f\|_{B M O}:=\sup _{I} \frac{1}{|I|} \int_{I}\left|f(s)-f_{I}\right| d s<\infty .
$$

The quantity $\|f\|_{B M O}$ is only a seminorm, since $\|f\|_{B M O}=0$ if $f$ equals a constant a.e. To turn BMO into a Banach space we can either restrict (2) to the linear space

$$
\left\{f \in L_{1}[0,1]: \int_{0}^{1} f(x) d x=0 \text { with }\|f\|_{B M O}<\infty\right\}
$$

(with identification of functions equal a.e.) or consider in $B M O$ one of the norms $\|f\|_{B M O}^{\prime}=\|f\|_{B M O}+\left|\int_{0}^{1} f(x) d x\right|$ or $\|f\|_{B M O}^{\prime \prime}=\|f\|_{B M O}+\|f\|_{L_{1}[0,1]}$.

We also introduce a dyadic version of $B M O$. If $I_{n}^{k}=\left(k / 2^{n},(k+1) / 2^{n}\right]$, $k=0,1, \ldots, 2^{n}-1, n=0,1,2, \ldots$, are dyadic intervals in $[0,1]$, then the space $B M O_{d}=B M O_{d}[0,1]$ consists of all $f \in L_{1}[0,1]$ such that

$$
\|f\|_{d}=\|f\|_{B M O_{d}}:=\sup _{k, n} \frac{1}{\left|I_{n}^{k}\right|} \int_{I_{n}^{k}}\left|f(s)-f_{I_{n}^{k}}\right| d s<\infty .
$$

It is clear that $B M O \subset B M O_{d}$ and $\|f\|_{d} \leq\|f\|_{B M O}$ for all $f \in B M O$. Moreover, $L_{\infty} \subset B M O$ and, for $f \in L_{\infty}[0,1]$, we have

$$
\begin{aligned}
\|f\|_{B M O} & \leq \sup _{I}\left(\frac{1}{|I|} \int_{I}\left(f(s)-f_{I}\right)^{2} d s\right)^{1 / 2} \\
& \leq \sup _{I}\left(\frac{1}{|I|} \int_{I}|f(s)|^{2} d s\right)^{1 / 2}=\|f\|_{L_{\infty}} .
\end{aligned}
$$

At the same time, $B M O \neq L_{\infty}$ and $B M O_{d} \neq B M O$. For example, we have $\ln |s-1 / 2| \chi_{[0,1]}(s) \in B M O \backslash L_{\infty}$ and $\ln |s-1 / 2| \chi_{[1 / 2,1]}(s) \in B M O_{d} \backslash B M O$.

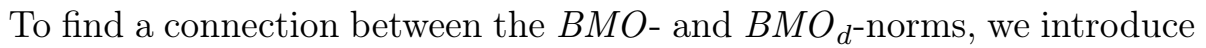
the functional

$$
A(f):=\sup _{I_{1}, I_{2}}\left|f_{I_{1}}-f_{I_{2}}\right|, \quad f \in L_{1}[0,1],
$$

where the supremum is taken over all adjacent dyadic intervals $I_{1}, I_{2}$ of the same length. 
The following assertion is an exercise from Garnett's book (cf. [8, Problem 12(b), p. 266]). We present the proof with concrete constants for the sake of completeness.

Proposition 1. For any $f \in L_{1}[0,1]$ we have

$$
\frac{1}{3}\left[\|f\|_{d}+A(f)\right] \leq\|f\|_{B M O} \leq 32\left[\|f\|_{d}+A(f)\right] .
$$

Proof. It is clear that the left-hand inequality of (3) is an immediate consequence of the estimate

$$
A(f) \leq 2\|f\|_{B M O} .
$$

To prove (4), take two adjacent dyadic intervals $I_{1}$ and $I_{2}$ of the same length. Then

$$
f_{I_{1} \cup I_{2}}=\frac{1}{2}\left(\frac{1}{\left|I_{1}\right|} \int_{I_{1}} f d s+\frac{1}{\left|I_{2}\right|} \int_{I_{2}} f d s\right)=\frac{1}{2}\left(f_{I_{1}}+f_{I_{2}}\right) .
$$

Therefore, for $I:=I_{1} \cup I_{2}$ we have

$$
\begin{aligned}
\frac{1}{\left|I_{1}\right|} \int_{I_{1}}\left|f-f_{I}\right| d s & =\frac{1}{2\left|I_{1}\right|} \int_{I_{1}}\left|\left(f-f_{I_{1}}\right)+\left(f-f_{I_{2}}\right)\right| d s \\
& \geq \frac{1}{2}\left|\frac{1}{\left|I_{1}\right|} \int_{I_{1}}\left(f-f_{I_{1}}\right) d s+\frac{1}{\left|I_{1}\right|} \int_{I_{1}}\left(f-f_{I_{2}}\right) d s\right| \\
& =\frac{1}{2}\left|f_{I_{1}}-f_{I_{2}}\right|,
\end{aligned}
$$

and similarly

$$
\frac{1}{\left|I_{2}\right|} \int_{I_{2}}\left|f-f_{I}\right| d s \geq \frac{1}{2}\left|f_{I_{1}}-f_{I_{2}}\right| .
$$

Thus,

$$
\begin{aligned}
\frac{1}{|I|} \int_{I}\left|f-f_{I}\right| d s & \left.=\frac{1}{2}\left(\frac{1}{\left|I_{1}\right|} \int_{I_{1}}\left|f-f_{I}\right| d s+\frac{1}{\left|I_{2}\right|} \int_{I_{2}} \mid f-f_{I}\right) \mid d s\right) \\
& \geq \frac{1}{2}\left|f_{I_{1}}-f_{I_{2}}\right|,
\end{aligned}
$$

which implies (4).

Let us prove the right-hand inequality of (3). For any $I \subset[0,1]$ we can find adjacent dyadic intervals $I_{1}$ and $I_{2}$ of the same length such that $I \subset I_{1} \cup I_{2}$ and

$$
\frac{1}{2}\left|I_{1}\right| \leq|I| \leq 2\left|I_{1}\right|
$$


Then

$$
\begin{aligned}
\frac{1}{|I|} \int_{I}\left|f(s)-f_{I}\right| d s & =\frac{1}{|I|} \int_{I}\left|f(s)-\frac{1}{|I|} \int_{I} f(t) d t\right| d s \\
& \leq \frac{1}{|I|^{2}} \iint_{I}|f(s)-f(t)| d t d s \\
& \leq \frac{16}{\left|I_{1} \cup I_{2}\right|^{2}} \int_{I_{1} \cup I_{2}} \int_{I_{1} \cup I_{2}}|f(s)-f(t)| d t d s \\
& \leq \frac{16}{\left|I_{1} \cup I_{2}\right|^{2}} \int_{I_{1} \cup I_{2}} \int_{I_{1} \cup I_{2}}\left|f(s)-f_{I_{1} \cup I_{2}}\right| d s d t \\
& +\frac{16}{\left|I_{1} \cup I_{2}\right|^{2}} \int_{I_{1} \cup I_{2}} \int_{I_{1} \cup I_{2}}\left|f(t)-f_{I_{1} \cup I_{2}}\right| d s d t \\
& =\frac{32}{\left|I_{1} \cup I_{2}\right|} \int_{I_{1} \cup I_{2}}\left|f(s)-f_{I_{1} \cup I_{2}}\right| d s .
\end{aligned}
$$

The above estimate and equality (5) imply that

$$
\begin{aligned}
\frac{1}{|I|} \int_{I}\left|f(s)-f_{I}\right| d s \leq & \frac{16}{\left|I_{1}\right|} \int_{I_{1}}\left|f(s)-\frac{1}{2}\left(f_{I_{1}}+f_{I_{2}}\right)\right| d s \\
& +\frac{16}{\left|I_{2}\right|} \int_{I_{2}}\left|f(s)-\frac{1}{2}\left(f_{I_{1}}+f_{I_{2}}\right)\right| d s \\
\leq & \frac{16}{\left|I_{1}\right|} \int_{I_{1}}\left|f(s)-f_{I_{1}}\right| d s+\frac{16}{\left|I_{2}\right|} \int_{I_{2}}\left|f(s)-f_{I_{2}}\right| d s \\
& +16\left|f_{I_{1}}-f_{I_{2}}\right| \leq 32\|f\|_{d}+16 A(f) .
\end{aligned}
$$

Hence,

$$
\|f\|_{B M O} \leq 32\|f\|_{d}+16 A(f) \leq 32\left[\|f\|_{d}+A(f)\right]
$$

3. Rademacher sums in $B M O$ spaces. The main purpose of this paper is to investigate the behaviour of Rademacher sums in the $B M O$ and $B M O_{d}$ spaces.

Proposition 2. For any $a_{k} \in \mathbb{R}, k=1, \ldots, n$, we have

$$
\frac{1}{\sqrt{2}}\left\|\left(a_{k}\right)_{k=1}^{n}\right\|_{l_{2}} \leq\left\|\sum_{k=1}^{n} a_{k} r_{k}\right\|_{d} \leq\left\|\left(a_{k}\right)_{k=1}^{n}\right\|_{l_{2}}
$$

and

$$
\frac{2}{3} \max _{0 \leq j<m \leq n}\left|\sum_{k=j+1}^{m} a_{k}\right| \leq A\left(\sum_{k=1}^{n} a_{k} r_{k}\right) \leq 4 \max _{0 \leq j<m \leq n}\left|\sum_{k=j+1}^{m} a_{k}\right| .
$$


Proof. Set $f=\sum_{k=1}^{n} a_{k} r_{k}$ and let $I$ be a dyadic interval of length $2^{-m}$, that is, $I=I_{m}^{i}=\left(i / 2^{m},(i+1) / 2^{m}\right]$. Then

$$
\left(r_{k}\right)_{I}= \begin{cases}\operatorname{sgn}\left(\left.r_{k}\right|_{I}\right) & \text { if } k \leq m, \\ 0 & \text { if } k>m,\end{cases}
$$

and so

$$
f_{I}=\sum_{k=1}^{n} a_{k}\left(r_{k}\right)_{I}=\sum_{k=1}^{\min (m, n)} a_{k} \operatorname{sgn}\left(\left.r_{k}\right|_{I}\right) .
$$

Thus, if $m \geq n$, then $\left.f\right|_{I}$ is a constant and therefore the oscillation of $f$ on $I$ vanishes, i.e., $O_{I}(f):=|I|^{-1} \int_{I}\left|f(x)-f_{I}\right| d x=0$. Otherwise, if $m<n$, we have

$$
\begin{aligned}
O_{I}(f) & =\frac{1}{|I|} \int_{I}\left|f(x)-f_{I}\right| d x=\frac{1}{|I|} \int\left|\sum_{I}{ }_{k=1}^{n} a_{k} r_{k}(x)-\sum_{k=1}^{m} a_{k} \operatorname{sgn}\left(\left.r_{k}\right|_{I}\right)\right| d x \\
& =\frac{1}{|I|} \int\left|\sum_{I=m+1}^{n} a_{k} r_{k}(x)\right| d x=\int_{0}^{1}\left|\sum_{k=m+1}^{n} a_{k} r_{k-m}(x)\right| d x \\
& =\left\|\sum_{k=m+1}^{n} a_{k} r_{k-m}\right\|_{L_{1}} .
\end{aligned}
$$

Using Khintchine's inequality (1) for the space $L_{1}[0,1]$ with the sharp constant $A_{1}=1 / \sqrt{2}$ (cf. [31]), we obtain

$$
\frac{1}{\sqrt{2}}\left(\sum_{k=m+1}^{n} a_{k}^{2}\right)^{1 / 2} \leq\left\|\sum_{k=m+1}^{n} a_{k} r_{k-m}\right\|_{L_{1}} \leq\left(\sum_{k=m+1}^{n} a_{k}^{2}\right)^{1 / 2} .
$$

Thus,

$$
\frac{1}{\sqrt{2}}\left(\sum_{k=1}^{n} a_{k}^{2}\right)^{1 / 2} \leq\left\|\sum_{k=1}^{n} a_{k} r_{k}\right\|_{d} \leq\left(\sum_{k=1}^{n} a_{k}^{2}\right)^{1 / 2} .
$$

Let now $I_{1}$ and $I_{2}$ be adjacent dyadic intervals of length $2^{-m}$ each. Then by the above observation,

$$
f_{I_{1}}-f_{I_{2}}=\sum_{k=1}^{\min (m, n)} a_{k} \operatorname{sgn}\left(\left.r_{k}\right|_{I_{1}}\right)-\sum_{k=1}^{\min (m, n)} a_{k} \operatorname{sgn}\left(\left.r_{k}\right|_{I_{2}}\right) .
$$

Let $I$ be the smallest dyadic interval containing $I_{1} \cup I_{2}$; let $I$ have length $2^{-j}$. Of course, $j<m$ and $\left.r_{k}\right|_{I_{1}}=\left.r_{k}\right|_{I_{2}}$ if $k \leq j$. Then for $j>n$ we have $f_{I_{1}}=f_{I_{2}}$, and for $j \leq n$,

$$
f_{I_{1}}-f_{I_{2}}=\sum_{k=j+1}^{\min (m, n)} a_{k}\left[\operatorname{sgn}\left(\left.r_{k}\right|_{I_{1}}\right)-\operatorname{sgn}\left(\left.r_{k}\right|_{I_{2}}\right)\right] .
$$


From the definition of $I$ it follows that $I_{1} \cup I_{2}$ is in the middle of $I$. Suppose that $I_{1}$ lies to the left of $I_{2}$. Then it is easy to see that $\left.r_{j+1}\right|_{I_{1}}=1,\left.r_{j+1}\right|_{I_{2}}=$ -1 and $\left.r_{k}\right|_{I_{1}}=-1,\left.r_{k}\right|_{I_{2}}=1$ if $j+2 \leq k \leq m$. Thus,

$$
\left|f_{I_{1}}-f_{I_{2}}\right|=2\left|\sum_{k=j+2}^{\min (m, n)} a_{k}-a_{j+1}\right|
$$

and

$$
A(f)=2 \max _{0 \leq j<m \leq n}\left|\sum_{k=j+2}^{m} a_{k}-a_{j+1}\right| .
$$

It is not hard to check that

$$
\frac{1}{3} \max _{0 \leq j<m \leq n}\left|\sum_{k=j+1}^{m} a_{k}\right| \leq \max _{0 \leq j<m \leq n}\left|\sum_{k=j+2}^{m} a_{k}-a_{j+1}\right| \leq 2 \max _{0 \leq j<m \leq n}\left|\sum_{k=j+1}^{m} a_{k}\right| .
$$

Combining this inequality with the previous equality, we obtain (7).

The following well-known assertion is an immediate consequence of inequalities (6) from Proposition 2. It was already proved by Garsia [10], [11] and even for martingale $B M O$ spaces. It was also obtained by Müller and Schechtman [25, Theorem 1] and Kochneff, Sagher and Zhou [16, Theorem 1], who also gave an example showing that the similar result for $B M O$ is not true.

THEOREM 1. The sequence $\left\{r_{k}\right\}_{k=1}^{\infty}$ of Rademacher functions is equivalent in $B M O_{d}$ to the standard unit basis in $l_{2}$.

From Propositions 1 and 2 and the elementary observation that

$$
\max _{1 \leq m \leq n}\left|\sum_{k=1}^{m} a_{k}\right| \leq \max _{0 \leq j<m \leq n}\left|\sum_{k=j+1}^{m} a_{k}\right| \leq 2 \max _{1 \leq m \leq n}\left|\sum_{k=1}^{m} a_{k}\right|,
$$

we obtain the following assertion:

Theorem 2. For any $a_{k} \in \mathbb{R}, k=1, \ldots, n$, and $n \in \mathbb{N}$ we have

$$
\begin{aligned}
\frac{2}{9}\left[\left(\sum_{k=1}^{n} a_{k}^{2}\right)^{1 / 2}+\max _{1 \leq m \leq n}\left|\sum_{k=1}^{m} a_{k}\right|\right] & \leq\left\|\sum_{k=1}^{n} a_{k} r_{k}\right\|_{B M O} \\
& \leq 256\left[\left(\sum_{k=1}^{n} a_{k}^{2}\right)^{1 / 2}+\max _{1 \leq m \leq n}\left|\sum_{k=1}^{m} a_{k}\right|\right] .
\end{aligned}
$$

In particular, the following equivalence holds:

$$
\left\|\sum_{k=1}^{\infty} a_{k} r_{k}\right\|_{B M O} \asymp\left\|\left(a_{k}\right)_{k=1}^{\infty}\right\|_{l_{2}}+\sup _{n \in \mathbb{N}}\left|\sum_{k=1}^{n} a_{k}\right| .
$$


COROLlary 1. The sequence $\left\{r_{k}\right\}_{k=1}^{\infty}$ of Rademacher functions is equivalent in BMO to each of its subsequences.

COROLlary 2. The system $\left\{r_{k}\right\}_{k=1}^{\infty}$ does not contain an unconditional basic subsequence in $B M O$.

For example, $\sum_{k=1}^{\infty}(-1)^{k} r_{k} / k \in \mathcal{R}$ and $\sum_{k=1}^{\infty} r_{k} / k \notin \mathcal{R}$.

Corollary 3. $L_{\infty}[0,1]$ is a unique (up to equivalence of norms) symmetric space on $[0,1]$ which is embedded into $B M O_{d}$.

Proof. For arbitrary $\delta \in(0,1)$ let us introduce the " $\delta$-translation" of the dyadic $B M O$, that is, the space $B M O_{d}(\delta)$ with the norm $\|f\|_{d, \delta}:=\left\|f_{\delta}\right\|_{d}$, where

$$
f_{\delta}(s):=f(s-\delta) \chi_{[\delta, 1]}(s)+f(s-\delta+1) \chi_{[0, \delta]}(s), \quad s \in[0,1] .
$$

Mei proved in [23] that there exists a $\delta_{0} \in(0,1)$ such that

$$
B M O=B M O_{d} \cap B M O_{d}\left(\delta_{0}\right) .
$$

If $X$ is a symmetric space on $[0,1]$ such that $X \subset B M O_{d}$, then $X \subset$ $B M O_{d}\left(\delta_{0}\right)$ as well. Thus, $X \subset B M O$. Next, since $\left\{r_{k}\right\}_{k=1}^{\infty}$ is an unconditional basic sequence with constant 1 in an arbitrary symmetric space (see, for example, [2, Corollary 1.7]), by Theorem 2 we obtain

$$
\left\|\sum_{k=1}^{\infty} a_{k} r_{k}\right\|_{X}=\left\|\sum_{k=1}^{\infty}\left|a_{k}\right| r_{k}\right\|_{X} \geq c_{1}\left\|\sum_{k=1}^{\infty}\left|a_{k}\right| r_{k}\right\|_{B M O} \geq c_{2}\left\|\left(a_{k}\right)\right\|_{l_{1}} .
$$

On the other hand, we have

$$
\left\|\sum_{k=1}^{\infty} a_{k} r_{k}\right\|_{X} \leq C\left\|\sum_{k=1}^{\infty} a_{k} r_{k}\right\|_{L_{\infty}}=C\left\|\left(a_{k}\right)\right\|_{l_{1}} .
$$

Therefore, $\left\{r_{k}\right\}_{k=1}^{\infty}$ is equivalent in $X$ to the standard unit basis in $l_{1}$ and, hence, by the Rodin-Semenov theorem [27, Theorem 7], we conclude that $X=L_{\infty}$ with equivalent norms.

COROLlary 4. The sequence $\left\{r_{k}\right\}_{k=1}^{\infty}$ of Rademacher functions is not weakly convergent to zero in BMO.

Proof. Define a linear functional on $\mathcal{R}$ by

$$
\varphi_{0}\left(\sum_{k=1}^{n} a_{k} r_{k}\right)=\sum_{k=1}^{n} a_{k}, \quad a_{k} \in \mathbb{R}, k=1, \ldots, n, n \in \mathbb{N} .
$$

Then, by the Hahn-Banach theorem, it can be extended to a functional $\varphi_{0} \in \mathcal{R}^{*}$, because in view of Theorem 2 we have

$$
\left|\varphi_{0}\left(\sum_{k=1}^{\infty} a_{k} r_{k}\right)\right|=\left|\sum_{k=1}^{\infty} a_{k}\right| \leq C|| \sum_{k=1}^{\infty} a_{k} r_{k} \|_{B M O} .
$$

Since $\varphi_{0}\left(r_{n}\right)=1 \nrightarrow 0$, we see that $r_{n} \nrightarrow 0$ weakly in $B M O$. 
REMARK 1. Astashkin and Maligranda 3] recently proved an equivalence completely similar to (8) for Cesàro function spaces $K_{p}$ on $[0,1]$ defined by the norms $\|f\|_{K_{p}}=\sup _{0<x \leq 1}\left(x^{-1} \int_{0}^{x}|f(t)|^{p} d t\right)^{1 / p}(1 \leq p<\infty)$ :

$$
\left\|\sum_{k=1}^{n} a_{k} r_{k}\right\|_{K_{p}} \asymp\left\|\left\{a_{k}\right\}_{k=1}^{n}\right\|_{l_{2}}+\max _{1 \leq m \leq n}\left|\sum_{k=1}^{m} a_{k}\right| .
$$

It is worth noting that the spaces $K_{p}$ and $B M O$ are not comparable, that is, neither is embedded in the other one.

\section{On complementability of Rademacher subspaces in $B M O$ and} $B M O_{d}$. We investigate the geometrical properties of the subspaces $\mathcal{R}_{d}:=$ $\left[r_{k}\right]_{B M O_{d}}$ and $\mathcal{R}:=\left[r_{k}\right]_{B M O}$ generated by the Rademacher system in $B M O_{d}$ and $B M O$, respectively. In fact, complementability of $\mathcal{R}_{d}$ in $B M O_{d}$ is wellknown (see, for example, Garsia [10], [11], Müller and Schechtman [25]). However, we present a simple proof.

TheOREM 3. The subspace $\mathcal{R}_{d}$ is complemented in $B M O_{d}$.

Proof. In view of a dyadic version of the John-Nirenberg theorem, which can be proved in the same way as the classical John-Nirenberg theorem (see, for example, [12, pp. 124-127]), for an arbitrary $f \in B M O_{d}$, any dyadic interval $I_{n}^{k}$ and $\tau>0$ we have

$$
m\left\{x \in I_{n}^{k}:\left|f(x)-f_{I_{n}^{k}}\right|>\tau\right\} \leq e\left|I_{n}^{k}\right| \exp \left(-\frac{\tau}{2 e\|f\|_{d}}\right) .
$$

This inequality implies that, for any $1 \leq p<\infty$,

$$
\|f\|_{d} \asymp\|f\|_{d, p}:=\sup _{k, n}\left(\frac{1}{\left|I_{n}^{k}\right|} \int_{I_{n}^{k}}\left|f(x)-f_{I_{n}^{k}}\right|^{p} d x\right)^{1 / p},
$$

with a constant depending on $p$ (see [12, p. 128] and [26, p. 525]). More precisely,

$$
\|f\|_{d} \leq\|f\|_{d, p} \leq 2 e[p \Gamma(p) e]^{1 / p}\|f\|_{d}
$$

Therefore, for every $1 \leq p<\infty$, we have $B M O_{d} \subset L_{p}[0,1]$. It is well-known that the orthogonal projector $P$ generated by the Rademacher system is bounded from $L_{p}[0,1]$ onto $\left[r_{k}\right]_{k=1}^{\infty}$ if $1<p<\infty$. Then, by (6) and the Khintchine inequality (1), for all $p \in(1, \infty)$ and $f \in B M O_{d}$,

$$
\|P f\|_{d} \asymp\|P f\|_{L_{p}} \leq\|P\|\|f\|_{L_{p}} \leq C_{p}\|P\|\|f\|_{d} .
$$

Thus, $P$ is bounded in $B M O_{d}$ and the proof is complete.

In contrast with Theorem 3 and with the remarkable theorem of Maurey 22] (cf. also [24, pp. 229-242]) which states that $B M O[0,1]$ is isomorphic to dyadic $B M O_{d}[0,1]$, the subspace $\mathcal{R}$ is an uncomplemented subspace of $B M O$. To prove this, we will need an auxiliary assertion. 
Denote by $\mathcal{U}=\left\{u_{n}\right\}_{n=1}^{\infty}$ a block basis of the Rademacher system, that is,

$$
u_{n}=\sum_{k=m_{n}+1}^{m_{n+1}} a_{k} r_{k} \quad(n=1,2, \ldots) \text {, }
$$

where $1 \leq m_{1}<m_{2}<\cdots$ and $a_{k} \in \mathbb{R}$. Moreover, let

$$
\gamma_{n}(\mathcal{U})=\sum_{k=m_{n}+1}^{m_{n+1}} a_{k}, \quad n=1,2, \ldots
$$

Proposition 3. The subspace $\mathcal{R}$ contains a complemented subspace $E$ isomorphic to $c_{0}$.

Proof. Take a block basis $\mathcal{U}=\left\{u_{n}\right\}_{n=1}^{\infty}$ satisfying the following assumptions:

(a) $\left\|u_{n}\right\|_{B M O}=1, n=1,2, \ldots$

(b) $\left\|u_{n}\right\|_{d} \asymp\left(\sum_{k=m_{n}+1}^{m_{n+1}} a_{k}^{2}\right)^{1 / 2} \leq 2^{-n}, n=1,2, \ldots$

(c) $\left|\gamma_{n}(\mathcal{U})\right| \leq 2^{-n}, n=1,2, \ldots$

It is clear that such a block basis exists. Let us prove that $\left[u_{n}\right]_{B M O}$ is isomorphic to $c_{0}$.

If $f=\sum_{n=1}^{\infty} \beta_{n} u_{n} \in \mathcal{R}$ with $\beta_{n} \in \mathbb{R}$, then

$$
f=\sum_{n=1}^{\infty}\left(\sum_{k=m_{n}+1}^{m_{n+1}} \beta_{n} a_{k} r_{k}\right)=\sum_{k=1}^{\infty} \gamma_{k} r_{k},
$$

where $\gamma_{k}=\beta_{n} a_{k}$ if $k=m_{n}+1, \ldots, m_{n+1}$. Assuming that $p, q \in \mathbb{N}$ satisfy

$$
m_{n-1} \leq p<m_{n}<m_{n+l}<q \leq m_{n+l+1}
$$

with some positive integers $n$ and $l$, we will estimate the sum $\sum_{k=p}^{q} \gamma_{k}$. Using (c), (a), Proposition 2 and inequality (4), we have

$$
\begin{aligned}
\left|\sum_{k=p}^{q} \gamma_{k}\right| & =\left|\sum_{k=p}^{m_{n}} \gamma_{k}+\sum_{k=m_{n}+1}^{m_{n+l}} \gamma_{k}+\sum_{k=m_{n+l}+1}^{q} \gamma_{k}\right| \\
& =\left|\sum_{k=p}^{m_{n}} \beta_{n-1} a_{k}+\sum_{i=n}^{n+l-1} \sum_{k=m_{i}+1}^{m_{i+1}} \beta_{i} a_{k}+\sum_{k=m_{n+l}+1}^{q} \beta_{n+l} a_{k}\right| \\
& \leq\left|\beta_{n-1}\right|\left|\sum_{k=p}^{m_{n}} a_{k}\right|+\sum_{i=n}^{n+l-1}\left|\beta_{i}\right|\left|\sum_{k=m_{i}+1}^{m_{i+1}} a_{k}\right|+\left|\beta_{n+l}\right|\left|\sum_{k=m_{n+l}+1}^{q} a_{k}\right|
\end{aligned}
$$




$$
\begin{aligned}
& \leq \sup _{n}\left|\beta_{n}\right|\left(\left|\sum_{k=p}^{m_{n}} a_{k}\right|+\sum_{i=n}^{n+l-1}\left|\sum_{k=m_{i}+1}^{m_{i+1}} a_{k}\right|+\left|\sum_{k=m_{n+l}+1}^{q} a_{k}\right|\right) \\
& \leq \sup _{n}\left|\beta_{n}\right|\left(\frac{3}{2} A\left(u_{n-1}\right)+\frac{3}{2} A\left(u_{n+l}\right)+\sum_{i=n}^{n+l-1} 2^{-i}\right) \\
& \leq\left(3\left\|u_{n-1}\right\|_{B M O}+3\left\|u_{n+l}\right\|_{B M O}+1\right)\left\|\left\{\beta_{n}\right\}\right\|_{c_{0}}=7\left\|\left\{\beta_{n}\right\}\right\|_{c_{0}} .
\end{aligned}
$$

Then from Theorem 2 and (b) it follows that $\|f\|_{B M O} \leq C\left\|\left\{\beta_{n}\right\}\right\|_{c_{0}}$.

On the other hand, by (a), (b) and Theorem 2, there is a $\delta>0$ such that $A\left(u_{n}\right) \geq \delta$ for all $n \in \mathbb{N}$. Therefore, by (4) and Proposition 2, we obtain

$$
\begin{aligned}
\|f\|_{B M O} & \geq \frac{1}{2} A(f) \geq \frac{1}{3} \sup _{n \in \mathbb{N} m_{n}+1 \leq p<q \leq m_{n+1}} \sup _{k=p}\left|\sum_{n}^{q} \beta_{n} a_{k}\right| \\
& \geq \frac{1}{12} \sup _{n \in \mathbb{N}}\left|\beta_{n}\right| A\left(u_{n}\right) \geq \frac{\delta}{12}\left\|\left\{\beta_{n}\right\}\right\|_{c_{0}} .
\end{aligned}
$$

Thus, we have proved that $E:=\left[u_{n}\right]_{B M O} \approx c_{0}$. Since $\mathcal{R}$ is separable, the Sobczyk theorem (see, for example, [1, Corollary 2.5.9]) implies that $E$ is a complemented subspace in $\mathcal{R}$.

TheOREM 4. The subspace $\mathcal{R}$ is not complemented in BMO.

Proof. Assume on the contrary that $\mathcal{R}$ is complemented in $B M O$ and let $P_{1}: B M O \rightarrow \mathcal{R}$ be a bounded linear projection whose range is $\mathcal{R}$. By Proposition 3, there is a subspace $E$ complemented in $\mathcal{R}$ and such that $E \approx c_{0}$. Let $P_{2}: \mathcal{R} \rightarrow E$ be a bounded linear projection. Then $P:=P_{2} \circ P_{1}$ is a linear projection bounded in $B M O$ with image $E$. Thus, $B M O$ contains a complemented subspace $E \approx c_{0}$. Since $B M O$ is a conjugate space (more precisely, $B M O=\left(\operatorname{Re} H_{1}\right)^{*}$ - see, for example, [15, p. 195]), this contradicts the well known result due to Bessaga-Pełczyński that a conjugate space cannot contain a complemented subspace isomorphic to $c_{0}$ (see [5, Corollary 4], which follows from Theorem 4 and its proof in [4]). This contradiction proves the theorem.

5. Structure of subspaces of $\mathcal{R}$. Sarason [29] introduced the $V M O=$ $V M O[0,1]$ space (space of vanishing mean oscillation in $[0,1]$ ) consisting of all $f \in B M O[0,1]$ for which $\lim _{|I| \rightarrow 0}|I|^{-1} \int_{I}\left|f(x)-f_{I}\right| d x=0$. This is a closed subspace of $B M O$ containing the space $C[0,1]$ of continuous functions and is equal to the $B M O$-closure of $C[0,1]$. The space $V M O$ was investigated by several authors. For example, it is known that $V M O$ is not complemented in $B M O$ (see, e.g., [13]). Structural properties of closed subspaces of $V M O$ were considered by Leibov [18, [19] who proved an analog of the Kadec-Pełczyński theorem for $V M O$ (Kadec-Pełczyński type alter- 
native [14]): an infinite-dimensional closed subspace of $V M O$ is either complemented in $B M O$ and isomorphic to $l_{2}$, or, for every $\varepsilon>0$, it contains a subspace which is complemented in $V M O$ and $(1+\varepsilon)$-isometric to $c_{0}$ (cf. [18, Theorem 3.4] and [19]). A similar dichotomy for the dyadic $V M O$ space was proved by Müller and Schechtman [25].

Our purpose here is to prove the Kadec-Pełczyński type alternative for the Rademacher subspace $\mathcal{R}$ of $B M O$.

TheOREM 5. Every infinite-dimensional closed subspace $X \subset \mathcal{R}$ is either isomorphic to $l_{2}$ and complemented in BMO, or contains a subspace $Y$ isomorphic to $c_{0}$ and complemented in $\mathcal{R}$.

In the proof of this theorem we will need some auxiliary result. Since $r_{n} \nrightarrow 0$ weakly in $B M O$ (cf. Corollary 4), it follows that the corresponding system of functionals biorthogonal to $\left\{r_{k}\right\}_{k=1}^{\infty}$ is not a basis of the space $\mathcal{R}^{*}$. Nevertheless, the following assertion holds.

Proposition 4. The space $\mathcal{R}^{*}$ has a basis.

Proof. Consider the sequence

$$
s_{n}=r_{n}-r_{n-1}, \quad n=1,2, \ldots, \quad \text { with } r_{0}=0 .
$$

If $f=\sum_{n=1}^{\infty} \beta_{n} s_{n}$, then

$$
f=\sum_{n=1}^{\infty} \beta_{n}\left(r_{n}-r_{n-1}\right)=\sum_{n=0}^{\infty}\left(\beta_{n}-\beta_{n+1}\right) r_{n}, \quad \text { where } \beta_{0}=0 .
$$

Therefore, by Theorem 2,

$$
\|f\|_{B M O} \asymp\left(\sum_{n=0}^{\infty}\left(\beta_{n}-\beta_{n+1}\right)^{2}\right)^{1 / 2}+\sup _{0 \leq m<n<\infty}\left|\beta_{m}-\beta_{n}\right|
$$

with a constant $c>0$. This implies, in particular, that

$$
\left|\beta_{n}\right| \leq\left|\beta_{n}-\beta_{1}\right|+\left|\beta_{1}\right| \leq 2 c\|f\|_{B M O}, \quad n=1,2, \ldots .
$$

Let us prove that $\left\{s_{n}\right\}_{n=1}^{\infty}$ is a shrinking basis in $\mathcal{R}$, that is, for any $\varphi \in \mathcal{R}^{*}$,

$$
\left\|\left.\varphi\right|_{\left[s_{n}\right]_{n=m}^{\infty}}\right\| \rightarrow 0 \quad \text { as } m \rightarrow \infty .
$$

Assume that (12) does not hold. Then there exist $\varepsilon \in(0,1)$, a functional $\varphi \in \mathcal{R}^{*}$ with $\|\varphi\|=1$, and a sequence of functions $f_{n}=\sum_{k=m_{n}}^{\infty} a_{k}^{m_{n}} s_{k}$, where $m_{1}<m_{2}<\cdots$, such that $\left\|f_{n}\right\|_{B M O}=1$ and $\varphi\left(f_{n}\right) \geq \varepsilon>0(n=1,2, \ldots)$.

We construct two sequences of positive integers, $\left\{q_{i}\right\}_{i=1}^{\infty}$ and $\left\{p_{i}\right\}_{i=1}^{\infty}$, $1<q_{1}<p_{1}<q_{2}<p_{2}<\cdots$, in the following way: $q_{1}=m_{1}$ and $p_{1}$ is chosen so that $\left\|\sum_{k=p_{1}+1}^{\infty} a_{k}^{q_{1}} s_{k}\right\|_{B M O}<\varepsilon / 2 ; q_{2}$ is the least $m_{n}>p_{1}$ and $p_{2}$ is such that $\left\|\sum_{k=p_{2}+1}^{\infty} a_{k}^{q_{2}} s_{k}\right\|_{B M O}<\varepsilon / 2 ; q_{3}$ is the least $m_{n}>p_{2}$ and $p_{3}$ is such that $\left\|\sum_{k=p_{3}+1}^{\infty} a_{k}^{q_{3}} s_{k}\right\|_{B M O}<\varepsilon / 2$; and so on. 
Set $a_{k}^{q_{i}}=0$ if $p_{i}<k<q_{i+1}, i=1,2, \ldots$ Then $\mathcal{U}=\left\{u_{i}\right\}$, where $u_{i}=\sum_{k=q_{i}}^{q_{i+1}-1} a_{k}^{q_{i}} s_{k}$, is a block basis of $\left\{s_{n}\right\}_{n=1}^{\infty}$. Moreover, by the definition of $u_{i}$,

$$
\sup _{i \in \mathbb{N}}\left\|u_{i}\right\|_{B M O} \leq 2
$$

and

$$
\varphi\left(u_{i}\right)=\varphi\left(f_{i}\right)-\varphi\left(\sum_{k=p_{i}+1}^{\infty} a_{k}^{q_{i}} s_{k}\right) \geq \varphi\left(f_{i}\right)-\left\|\sum_{k=p_{i}+1}^{\infty} a_{k}^{q_{i}} s_{k}\right\|_{B M O} \geq \frac{\varepsilon}{2} .
$$

Let us show that for every nonnegative sequence $\left\{\gamma_{n}\right\}_{n=1}^{\infty}$ such that

$$
\sum_{n=1}^{\infty} \gamma_{n}^{2}<\infty \text { and } \sum_{n=1}^{\infty} \gamma_{n}=\infty
$$

the series $\sum_{n=1}^{\infty} \gamma_{n} u_{n}$ converges in $B M O$.

Let $b_{k}=a_{k}^{q_{i}} \gamma_{i}$ for $q_{i} \leq k<q_{i+1}, i=1,2, \ldots$ If $q_{i} \leq k<q_{i+1}$, then, in view of (11) and (13), we obtain $\left|b_{k}\right| \leq 4 c \gamma_{i}$, and hence

$$
\lim _{k \rightarrow \infty} b_{k}=0 \text {. }
$$

Moreover,

$$
\begin{aligned}
\sum_{k=1}^{\infty}\left(b_{k}-b_{k+1}\right)^{2}= & \sum_{i=1}^{\infty} \sum_{k=q_{i}}^{q_{i+1}-2}\left(a_{k}^{q_{i}} \gamma_{i}-a_{k+1}^{q_{i}} \gamma_{i}\right)^{2} \\
& +\sum_{i=1}^{\infty}\left(a_{q_{i+1}-1}^{q_{i}} \gamma_{i}-a_{q_{i+1}}^{q_{i+1}} \gamma_{i+1}\right)^{2}=A_{1}+A_{2} .
\end{aligned}
$$

We will estimate $A_{1}$ and $A_{2}$ separately. By 10 ,

$$
A_{1}=\sum_{i=1}^{\infty} \gamma_{i}^{2} \sum_{k=q_{i}}^{q_{i+1}-2}\left(a_{k}^{q_{i}}-a_{k+1}^{q_{i}}\right)^{2} \leq c^{2} \sum_{i=1}^{\infty} \gamma_{i}^{2}
$$

and, by 11,

$$
\begin{aligned}
A_{2} & \leq 2 \sum_{i=1}^{\infty}\left(\left(a_{q_{i+1}-1}^{q_{i}}\right)^{2} \gamma_{i}^{2}+\left(a_{q_{i+1}}^{q_{i+1}}\right)^{2} \gamma_{i+1}^{2}\right) \\
& \leq 4 \sup _{i \in \mathbb{N}} \max _{q_{i} \leq k<q_{i+1}}\left|a_{k}^{q_{i}}\right|^{2} \sum_{i=1}^{\infty} \gamma_{i}^{2} \leq 16 c^{2} \sum_{i=1}^{\infty} \gamma_{i}^{2} .
\end{aligned}
$$

Therefore, according to 10 and 15 , the series

$$
\sum_{n=1}^{\infty} \gamma_{n} u_{n}=\sum_{k=1}^{\infty} b_{k} s_{k}
$$


converges in $B M O$. On the other hand, taking into account (14), we have

$$
\varphi\left(\sum_{n=1}^{\infty} \gamma_{n} u_{n}\right)=\sum_{n=1}^{\infty} \gamma_{n} \varphi\left(u_{n}\right) \geq \frac{\varepsilon}{2} \sum_{n=1}^{\infty} \gamma_{n}=\infty,
$$

which contradicts $\varphi \in \mathcal{R}^{*}$. Thus, $(12)$ is proved.

Finally, by Proposition 1.b.1 in [20], the biorthogonal system $\left\{s_{n}^{*}\right\}_{n=1}^{\infty}$ of functionals is a basis in the space $\mathcal{R}^{*}$ and the proof is complete.

Proof of Theorem 5. Assume that there is an $\varepsilon>0$ such that $\|f\|_{d} \geq$ $\varepsilon\|f\|_{B M O}$ for every $f \in X$. Then, according to (6) and (1), for every $1 \leq p$ $<\infty$ and all $f=\sum_{k=1}^{\infty} a_{k} r_{k} \in X$,

$$
\|f\|_{B M O} \leq \varepsilon^{-1}\|f\|_{d} \leq \varepsilon^{-1}\left(\sum_{k=1}^{\infty} a_{k}^{2}\right)^{1 / 2} \leq \varepsilon^{-1} A_{p}^{-1}\|f\|_{L_{p}[0,1]} .
$$

On the other hand, $B M O \subset L_{p}$ for every $1 \leq p<\infty$ (see the proof of Theorem 3). Therefore, the $B M O$-norm on $X$ is equivalent to the $L_{p}$-norm for every $1 \leq p<\infty$. In particular, this implies that $X$ is isomorphic to $l_{2}$. Since the subspace $\mathcal{R}_{p}$ generated by the Rademacher system is complemented in $L_{p}$ if $1<p<\infty$, and $\mathcal{R}_{p}$ is isomorphic to $l_{2}$, it follows that $X$ is complemented in $L_{p}$ as well. Denote by $P$ a linear projection bounded from $L_{p}$ onto $X$. Then, by (16),

$$
\begin{aligned}
\|P f\|_{B M O} & \leq \varepsilon^{-1} A_{p}^{-1}\|P f\|_{L_{p}} \leq A_{p}^{-1} \varepsilon^{-1}\|P\|_{L_{p} \rightarrow L_{p}}\|f\|_{L_{p}} \\
& \leq C_{p}(\varepsilon)\|P\|_{L_{p} \rightarrow L_{p}}\|f\|_{B M O} .
\end{aligned}
$$

Therefore, $X$ is complemented in $B M O$.

Now, assume that there is a sequence $\left\{f_{n}\right\}_{n=1}^{\infty} \subset X$ with $\left\|f_{n}\right\|_{B M O}=1$ $(n=1,2, \ldots)$ such that $\left\|f_{n}\right\|_{d} \rightarrow 0$ as $n \rightarrow \infty$. Then $\left\{f_{n}\right\}_{n=1}^{\infty}$ does not contain any subsequence converging in $B M O$. In fact, if $\lim _{k \rightarrow \infty} f_{n_{k}}=f \in$ $B M O$ for some $\left\{f_{n_{k}}\right\} \subset\left\{f_{n}\right\}$, then we have both $\|f\|_{B M O}=1$ and $\|f\|_{d}=0$ (and therefore $f=0$ ), which is impossible.

Hence, passing to a subsequence if necessary, we may assume that for some $\delta>0$,

$$
\left\|f_{m}-f_{n}\right\|_{B M O} \geq \delta>0 \quad \text { for all } m, n=1,2, \ldots, m \neq n .
$$

Let $\left\{s_{k}^{*}\right\}_{k=1}^{\infty}$ be the basis of $\mathcal{R}^{*}$ constructed in the proof of Proposition 4 . Using the diagonal process, it is not hard to choose a subsequence $\left\{f_{n_{i}}\right\}_{i=1}^{\infty} \subset$ $\left\{f_{n}\right\}$ such that for any $k \in \mathbb{N}$ we have

$$
s_{k}^{*}\left(f_{n_{i+1}}-f_{n_{i}}\right) \rightarrow 0 \quad \text { as } i \rightarrow \infty .
$$

Since $\left\{s_{k}^{*}\right\}_{k=1}^{\infty}$ is a basis of $\mathcal{R}^{*}$ and $\left\|f_{n}\right\|_{B M O}=1(n=1,2, \ldots)$, it follows that

$$
f_{n_{i+1}}-f_{n_{i}} \rightarrow 0 \quad \text { weakly in } B M O
$$


Then, by (17) and by Proposition 1.a.12 in [20, there is a block basis $\mathcal{U}=$ $\left\{u_{k}\right\}_{k=1}^{\infty}$ of the Rademacher system, which is equivalent to some subsequence of $\left\{f_{n_{i+1}}-f_{n_{i}}\right\}_{i=1}^{\infty}$ (denoted $\left\{f_{n_{i+1}}-f_{n_{i}}\right\}$ as well). In particular,

$$
\left\|u_{k}-\left(f_{n_{k+1}}-f_{n_{k}}\right)\right\|_{B M O} \leq 2^{-k}, \quad k=1,2, \ldots .
$$

This implies that both $u_{k} \rightarrow 0$ weakly in $B M O$ and $\left\|u_{k}\right\|_{d} \rightarrow 0$ as $k \rightarrow \infty$.

Let $\varphi_{0} \in \mathcal{R}^{*}$ be as in the proof of Corollary 4. If $u_{k}=\sum_{i=m_{k}+1}^{m_{k+1}} a_{i} r_{i}$, $1 \leq m_{1}<m_{2}<\cdots$, then

$$
\varphi_{0}\left(u_{k}\right)=\sum_{i=m_{k}+1}^{m_{k+1}} a_{i} \rightarrow 0 \quad \text { as } k \rightarrow \infty .
$$

Therefore, using the same arguments as in the proof of Proposition 3, we may choose a subsequence $\left\{u_{k_{i}}\right\}_{i=1}^{\infty}$ such that $\left[u_{k_{i}}\right]_{i=1}^{\infty} \approx c_{0}$ and $\left[u_{k_{i}}\right]_{i=1}^{\infty}$ is complemented in $\mathcal{R}$.

Using Theorem 5, we are able to describe the structure of subspaces of $\mathcal{R}$ in the following way.

Corollary 5. Let $X \subset \mathcal{R}$ be an infinite-dimensional closed subspace of BMO. The following conditions are equivalent:

(1) $X$ does not contain a subspace isomorphic to $c_{0}$.

(2) $X$ is isomorphic to a dual space.

(3) $X$ is reflexive.

(4) $X$ is isomorphic to $l_{2}$.

(5) $X$ is complemented in $B M O$.

(6) The BMO-norm on $X$ is equivalent to the $L_{1}$-norm.

Proof. By Theorem 5, condition (1) implies either of conditions (2)-(6). Conversely, it is obvious that each of the conditions (3), (4) and (6) implies (1). The implications $(2) \Rightarrow(1)$ and $(5) \Rightarrow(1)$ are consequences of the above mentioned results of Bessaga-Pełczyński.

Recall that the function $f_{\delta}$ was defined in 9 .

Corollary 6 . There is a $\delta \in(0,1)$ such that no bounded linear projection $P$ in $B M O_{d}$ with range $\mathcal{R}_{d}$ commutes with the $\delta$-shift on $K_{\delta}:=$ $B M O_{d} \cap B M O_{d}(\delta)$. This means that for every such projection there is a function $f \in K_{\delta}$ such that $P\left(f_{\delta}\right) \neq(P f)_{\delta}$.

Proof. Suppose, on the contrary, that for any $\delta \in(0,1)$ there exists a bounded linear projection $P: B M O_{d} \rightarrow \mathcal{R}_{d}$ such that $P\left(f_{\delta}\right)=(P f)_{\delta}$ for every $f \in K_{\delta}$. By Mei's theorem (cf. [23]) there is $\delta_{0} \in(0,1)$ such that $K_{\delta_{0}}=B M O$ and

$$
\|f\|_{B M O} \asymp \max \left(\|f\|_{d},\|f\|_{d, \delta_{0}}\right) .
$$


Then for any $f \in B M O$, by assumption,

$$
\begin{aligned}
\|P f\|_{B M O} & \asymp \max \left(\|P f\|_{d},\|P f\|_{d, \delta_{0}}\right) \leq \max \left(C\|f\|_{d},\left\|(P f)_{\delta_{0}}\right\|_{d}\right) \\
& =\max \left(C\|f\|_{d},\left\|P\left(f_{\delta_{0}}\right)\right\|_{d}\right) \leq C \max \left(\|f\|_{d},\left\|f_{\delta_{0}}\right\|_{d}\right) \\
& =C \max \left(\|f\|_{d},\|f\|_{d, \delta_{0}}\right) \asymp\|f\|_{B M O},
\end{aligned}
$$

which implies that $P$ is bounded in $B M O$, contrary to Theorem 4 .

To end the paper, we consider some examples of block bases of the Rademacher system whose span is $l_{2}$ and $c_{0}$, respectively.

EXAMPLE 1. A block basis of the Rademacher system which spans $l_{2}$ in $B M O$. Let $u_{k}:=r_{2 k+1}-r_{2 k}$ and $f=\sum_{k=1}^{n} a_{k} u_{k}, n=1,2, \ldots$ Then

$$
f=\sum_{k=1}^{n} a_{k}\left(r_{2 k+1}-r_{2 k}\right)=\sum_{k=1}^{n} a_{k} r_{2 k+1}-\sum_{k=1}^{n} a_{k} r_{2 k}
$$

and, by (7),

$$
A(f) \asymp \max _{1 \leq k \leq n}\left|a_{k}\right|=\left\|\left\{a_{k}\right\}_{k=1}^{n}\right\|_{c_{0}} .
$$

On the other hand, according to (6),

$$
\frac{1}{\sqrt{2}}\left\|\left\{a_{k}\right\}_{k=1}^{n}\right\|_{l_{2}} \leq\|f\|_{d} \leq 2\left\|\left\{a_{k}\right\}_{k=1}^{n}\right\|_{l_{2}} .
$$

Combining these relations with Theorem 2, we obtain

$$
\left\|\sum_{k=1}^{n} a_{k} u_{k}\right\|_{B M O} \asymp\left\|\left\{a_{k}\right\}_{k=1}^{n}\right\|_{l_{2}}, \quad n=1,2, \ldots
$$

EXAMPle 2. A block basis of the Rademacher system which spans $c_{0}$ in $B M O$. Take a block basis $\mathcal{U}=\left\{u_{n}\right\}_{n=1}^{\infty}$, where

$$
u_{n}=\sum_{k=m_{n}+1}^{m_{n+1}} a_{k} r_{k} \quad \text { with } \quad m_{n+1}-m_{n}=2^{2 n}, \quad n=1,2, \ldots,
$$

and

$$
a_{k}= \begin{cases}2^{-2 n} & \text { if } m_{n}+1 \leq k \leq\left(m_{n}+m_{n+1}\right) / 2, \\ -2^{-2 n} & \text { if }\left(m_{n}+m_{n+1}\right) / 2+1 \leq k \leq m_{n+1} .\end{cases}
$$

Then, by (6) and (8),

$$
\left\|u_{n}\right\|_{d} \asymp\left(\sum_{k=m_{n}+1}^{m_{n+1}} 2^{-4 n}\right)^{1 / 2}=2^{-n}, \quad n=1,2, \ldots,
$$

and

$$
\left\|u_{n}\right\|_{B M O} \asymp 2^{-2 n} \frac{m_{n+1}-m_{n}}{2}+2^{-n} \asymp \frac{1}{2}, \quad n=1,2, \ldots
$$


Moreover,

$$
\gamma_{n}(\mathcal{U})=\sum_{k=m_{n}+1}^{\left(m_{n}+m_{n+1}\right) / 2} 2^{-2 n}-\sum_{k=\left(m_{n}+m_{n+1}\right) / 2+1}^{m_{n+1}} 2^{-2 n}=0 .
$$

Then (see the proof of Proposition 3) $\left[u_{n}\right]_{B M O} \approx c_{0}$.

Acknowledgements. Research of S. V. Astashkin was partially supported by RFBR grant no. 10-01-00077.

Research of L. Maligranda was partially supported by the Swedish Research Council (VR) grant 621-2008-5058.

\section{References}

[1] F. Albiac and N. J. Kalton, Topics in Banach Space Theory, Grad. Texts in Math. 233, Springer, New York, 2006.

[2] S. V. Astashkin, Rademacher functions in symmetric spaces, Sovrem. Mat. Fundam. Napravl. 32 (2009), 3-161 (in Russian); English transl.: J. Math. Sci. (New York) 169 (2010), 725-886.

[3] S. V. Astashkin and L. Maligranda, Rademacher functions in Cesàro type spaces, Studia Math. 198 (2010), 235-247.

[4] C. Bessaga and A. Pełczyński, On bases and unconditional convergence of series in Banach spaces, ibid. 17 (1958), 151-164.

[5] -, -, Some remarks on conjugate spaces containing subspaces isomorphic to the space $c_{0}$, Bull. Acad. Polon. Sci. Sér. Sci. Math. Astronom. Phys. 6 (1958), 249-250.

[6] C. Fefferman, Characterizations of bounded mean oscillation, Bull. Amer. Math. Soc. 77 (1971), 587-588.

[7] C. Fefferman and E. M. Stein, $H^{p}$ spaces of several variables, Acta Math. 129 (1972), $137-193$.

[8] J. B. Garnett, Bounded Analytic Functions, Springer, New York, 2007.

[9] J. B. Garnett and P. W. Jones, BMO from dyadic BMO, Pacific J. Math. 99 (1982), 351-371.

[10] A. M. Garsia, The Burgess Davis inequalities via Fefferman's inequality, Ark. Mat. 11 (1973), 229-237.

[11] -, Martingale Inequalities: Seminar Notes on Recent Progress, Benjamin, Reading, MA, 1973.

[12] L. Grafakos, Modern Fourier Analysis, 2nd ed., Springer, New York, 2009.

[13] D. Hadwin and H. Yousefi, A general view of BMO and VMO, in: Contemp. Math. 454, Amer. Math. Soc., Providence, 2008, 75-91.

[14] M. I. Kadec and A. Pełczyński, Bases, lacunary sequences and complemented subspaces in the spaces $L_{p}$, Studia Math. 21 (1962), 161-176.

[15] B. S. Kashin and A. A. Saakyan, Orthogonal Series, Nauka, Moscow, 1984 (in Russian); English transl.: Amer. Math. Soc., Providence, 1989.

[16] E. Kochneff, Y. Sagher and K. C. Zhou, BMO estimates for lacunary series, Ark. Mat. 28 (1990), 301-310. 
[17] S. G. Kreı̆n, Yu. I. Petunin, and E. M. Semenov, Interpolation of Linear Operators, Nauka, Moscow, 1978 (in Russian); English transl.: Amer. Math. Soc., Providence, 1982.

[18] M. V. Leibov, The Geometry of the Function Space BMO, Candidate's Dissertation, Rostov-na-Donu, 1985, 133 pp. (in Russian).

[19] —, Subspaces of the space VMO, Teor. Funktsii Funktsional. Anal. i Prilozhen. 46 (1986), 51-54 (in Russian); English transl.: J. Soviet Math. 48 (1990), 536-538.

[20] J. Lindenstrauss and L. Tzafriri, Classical Banach Spaces, I. Sequence Spaces, Springer, Berlin, 1977.

[21] -, -, Classical Banach Spaces, II. Function Spaces, Springer, Berlin, 1979.

[22] B. Maurey, Isomorphismes entre espaces $H_{1}$, Acta Math. 145 (1980), 79-120.

[23] T. Mei, BMO is the intersection of two translates of dyadic BMO, C. R. Math. Acad. Sci. Paris 336 (2003), 1003-1006.

[24] P. F. Müller, Isomorphisms between $H^{1}$ Spaces, IMPAN Monogr. Mat. 66, Birkhäuser, Basel, 2005.

[25] P. F. Müller and G. Schechtman, On complemented subspaces of $H^{1}$ and VMO, in: Lecture Notes in Math. 1376, Springer, Berlin, 1989, 113-125.

[26] J. Pipher and L. A. Ward, BMO from dyadic BMO on the bidisc, J. London Math. Soc. (2) 77 (2008), 524-544.

[27] V. A. Rodin and E. M. Semyonov, Rademacher series in symmetric spaces, Anal. Math. 1 (1975), 207-222.

[28] - - - The complementability of a subspace that is generated by the Rademacher system in a symmetric space, Funktsional. Anal. i Prilozhen. 13 (1979), no. 2, 91-92 (in Russian); English transl.: Funct. Anal. Appl. 13 (1979), no. 2, 150-151.

[29] D. Sarason, Functions of vanishing mean oscillation, Trans. Amer. Math. Soc. 207 (1975), 391-405.

[30] E. M. Stein, Harmonic Analysis: Real-Variable Methods, Orthogonality, and Oscillatory Integrals, Princeton Univ. Press, Princeton, 1993.

[31] S. J. Szarek, On the best constants in the Khinchin inequality, Studia Math. 58 (1976), 197-208.

Sergey V. Astashkin

Department of Mathematics and Mechanics

Samara State University

Akad. Pavlova 1

443011 Samara, Russia

E-mail: astashkn@ssu.samara.ru

Lech Maligranda

Department of Engineering Sciences and Mathematics

Luleå University of Technology

SE-971 87 Luleå, Sweden

E-mail: lech.maligranda@ltu.se
Mikhail Leibov

Horton Point LLC

1180 Avenue of the Americas New York, NY 10036, U.S.A.

E-mail: mleybov@gmail.com

Received April 3, 2011

Revised version June 9, 2011 